ARTICLE

\title{
Flux periodic oscillations and phase-coherent transport in GeTe nanowire-based devices
}

Jinzhong Zhang1,2,3, Pok-Lam Tse (10) 4, Abdur-Rehman Jalil (10) 1,2, Jonas Kölzer 1,2, Daniel Rosenbach,2, Martina Luysberg ${ }^{5}{ }^{5}$, Gregory Panaitov ${ }^{6}$, Hans Lüth', ${ }^{1,2}$ Zhigao $\mathrm{Hu}^{3}$, Detlev Grützmacher ${ }^{1,2}$, Jia Grace $\mathrm{Lu}^{4} \&$ Thomas Schäpers (iD) 1,2凶

Despite the fact that GeTe is known to be a very interesting material for applications in thermoelectrics and for phase-change memories, the knowledge on its low-temperature transport properties is only limited. We report on phase-coherent phenomena in the magnetotransport of GeTe nanowires. From universal conductance fluctuations measured on GeTe nanowires with Au contacts, a phase-coherence length of about $280 \mathrm{~nm}$ at $0.5 \mathrm{~K}$ is determined. The distinct phase-coherence is confirmed by the observation of Aharonov-Bohm type oscillations for parallel magnetic fields. We interpret the occurrence of these magnetic flux-periodic oscillations by the formation of a tubular hole accumulation layer. For $\mathrm{Nb} / \mathrm{GeTe}$-nanowire/Nb Josephson junctions we obtained a critical current of $0.2 \mu \mathrm{A}$ at $0.4 \mathrm{~K}$. By applying a perpendicular magnetic field the critical current decreases monotonously with increasing field, whereas in a parallel field the critical current oscillates with a period of the magnetic flux quantum confirming the presence of a tubular hole channel.

\footnotetext{
${ }^{1}$ Peter Grünberg Institut (PGI-9), Forschungszentrum Jülich, Jülich 52425, Germany. ${ }^{2}$ JARA-Fundamentals of Future Information Technology, Jülich-Aachen Research Alliance, Forschungszentrum Jülich and RWTH Aachen University, Jülich 52425, Germany. ${ }^{3}$ Technical Center for Multifunctional Magneto-Optical Spectroscopy (Shanghai), Engineering Research Center of Nanophotonics \& Advanced Instrument (Ministry of Education), Department of Materials, School of Physics and Electronics Science, East China Normal University, Shanghai 200241, China. ${ }^{4}$ Department of Physics and Astronomy and Department of Electrophysics, University of Southern California, Los Angeles 90089 CA, USA. ${ }^{5}$ Ernst Ruska Center, Forschungszentrum Jülich, Jülich 52425, Germany.

${ }^{6}$ Institute of Complex Systems (ICS-8) Forschungszentrum Jülich, Jülich 52425, Germany. ${ }^{凶}$ email: th.schaepers@fz-juelich.de
} 
$\mathrm{n}$ the past decades, GeTe-based alloys attracted an increasing interest for their applications in optical data storage and phase change memories due to a reversible rapid transformation between amorphous and crystalline phase ${ }^{1,2}$, and a large contrast of optical constants and electrical conductivity for the two pha$\operatorname{ses}^{3}$. In addition, GeTe-rich alloys can be used in intermediate temperature thermoelectric applications for converting waste heat to electrical energy because of their high thermoelectric performance, high thermal and mechanical stability ${ }^{4,5}$. From the fundamental physics perspective it is important to unravel its intrinsic property governed by the unique metavalent bonding and to shed light on the microscopic mechanisms that contribute to its phase change property ${ }^{6}$.

Pristine GeTe is a p-type semiconductor with a trigonal ferroelectric phase (space group $R 3 m$, No. 160) and an indirect band gap of $E_{\mathrm{g}} \sim 0.61 \mathrm{eV}^{4,7-9}$. Only recently it was recognized that its trigonal ferroelectric phase makes this material very attractive for spinelectronic applications ${ }^{8}$. This is due to the fact that the noncentrosymmetric structure with displaced adjacent $\mathrm{Ge}$ and $\mathrm{Te}$ layers along the [111] direction exhibit a net dielectric polarization. The inversion symmetry breaking as a result of the displacement leads to a giant Rashba spin splitting of the bulk bands in $\mathrm{GeTe}^{8,10-12}$. In fact, photoemission spectroscopy measurements confirmed the Rashba band splitting as well as the corresponding spin polarization ${ }^{13-16}$. As a further verification of the presence of Rashba spin-orbit interaction, weak antilocalization was observed in GeTe layers in low-temperature magnetotransport experiments. In addition to spin-related properties GeTe is observed to be superconducting at temperatures below $0.3 \mathrm{~K}^{17-19}$. Recently, Narayan et al. ${ }^{20}$ have investigated the non-equilibrium superconductivity in GeTe Hall bar samples with a semiconductor-superconductor transition temperature of about $0.14 \mathrm{~K}$ and a critical magnetic field of around $70 \mathrm{mT}$. Last but not least, the combination of strong spin-orbit coupling and superconductivity makes GeTe a very attractive candidate for topological systems ${ }^{19,21}$.

To explore possible device applications the transport properties of quasi one-dimensional GeTe nanowires have been investigated. Here, it turned out that GeTe nanowires are very interesting as building blocks in phase-change memories ${ }^{22-24}$. However, to find out about the suitability of GeTe for the above mentioned spintronic and quantum device applications low-temperature quantum transport measurements on nanostructures are required. In order to do so, we have grown GeTe nanowires and measured the transport properties of GeTe nanowires contacted with normal metals as well as GeTe nanowire-based Josephson junctions. For the normal contacted nanowires we investigated phase-coherent transport phenomena such as universal conductance fluctuations, weak antilocalization, as well as Aharonov-Bohm-type resistance oscillations. For the GeTe nanowires equipped with $\mathrm{Nb}$ contacts the proximity-induced superconductivity as well as critical temperature and magnetic field of $\mathrm{Nb} / \mathrm{GeTe}$-nanowire/ $\mathrm{Nb}$ Josephson junctions are studied.

\section{Results and discussion}

GeTe nanowires (NWs) were synthesized by a Au-catalyzed vapor-liquid-solid growth in a tube furnace with GeTe powders 22,25 . The as-grown $\mathrm{GeTe} \mathrm{NWs}$ on a $\mathrm{Si} / \mathrm{SiO}_{2}$ substrate are straight with a length of more than $5 \mu \mathrm{m}$. The cross section for the GeTe nanowires has a rhombic shape with a side length of about $80 \mathrm{~nm}$ (cf. Fig. 1a). A high-angle annular dark field (HAADF) image of GeTe NW cross section was obtained via aberration corrected scanning transmission electron microscopy (STEM). In the corresponding image shown in Fig. 1b one can identify a trigonal crystal structure. The positions of the Te and Ge atoms are indicated by light green and magenta dots, respectively. For device fabrication the GeTe NWs were transferred to a $\mathrm{Si} / \mathrm{SiO}_{2}$ substrate. Subsequently, superconducting $(\mathrm{Nb})$ or normal-metal (Ti/Au) contact electrodes were prepared by using electron beam lithography and magnetic sputtering.

In order to investigate the material properties of the nanowires energy dispersive X-ray spectroscopy (EDX) and Raman spectroscopy were performed. The EDX results shown in Fig. 1c reveal that the as-grown GeTe has an ideal atomic ratio of Ge:Te $=1: 1$ and the element component are uniform along the NWs except the nanowire ends with a Au nanoparticle, typical of vapor-liquid-solid growth. Furthermore, Raman spectra of a GeTe single nanowire demonstrate unambiguously that GeTe NWs exhibit rhombohedral phase $(R 3 m)$ at room temperature (cf. Fig. 1d). According to the group theory, the two peaks near 89 and $119 \mathrm{~cm}^{-1}$ are assigned to the first-order Raman-active modes $E(\mathrm{TO})$ and $A_{1}(\mathrm{LO})$, respectively ${ }^{26,27}$. Theoretically, $E$ and $A_{1}$ relates to the bending modes of the $\mathrm{GeTe}_{4}$ tetrahedra and symmetric stretching vibration of the $\mathrm{Te}-\mathrm{Te}$ bond, respectively ${ }^{28}$. Note that the peak labeled by the symbol $\left(^{*}\right)$ at around $140 \mathrm{~cm}^{-1}$ arises from surface oxidation ${ }^{29}$. Other peaks/shoulders may originate from the breaking of inversion symmetry due to vacancies, defects, and distortion of bonds ${ }^{30}$.

In order to gain information on the electrical properties of the GeTe nanowires we measured the magnetotransport of samples equipped with normal contacts (Ti/Au: $20 \mathrm{~nm} / 100 \mathrm{~nm}$ ). An electron beam micrograph of the contacted 80 -nm-wide nanowire is shown in Fig. 2a (inset). The measurements were performed in a four-terminal configuration with the bias current supplied at the outer contacts. The width and separation of the inner electrodes is about 0.5 and $1.0 \mu \mathrm{m}$, respectively. In Fig. $2 \mathrm{a}$ the resistance as a function of temperature is plotted. One finds that the resistance decreases with decreasing temperature in the temperature range from 35 to $10 \mathrm{~K}$, and then increases monotonously upon further decrease of the temperature down to $0.4 \mathrm{~K}$. The temperature dependence corresponds to a metallic behavior, where the upturn in resistance for temperatures below $10 \mathrm{~K}$ can be attributed to the electron-electron interaction and weak localization effects ${ }^{31-33}$. No transition to a superconducting behavior is observed, probably because the lowest temperature of $0.4 \mathrm{~K}$ is larger than the critical temperature ${ }^{17,19}$.

Figure $2 \mathrm{~b}$ shows the conductance $G$ in units of $e^{2} / h$ with the magnetic field applied perpendicular to the nanowire axis $\left(B_{\perp}\right.$, $\theta=90^{\circ}$ ), at temperatures between 0.5 and $5.0 \mathrm{~K}$. Here, $e$ is the electron charge and $h$ is the Planck constant. Similarly to GeTe films ${ }^{19}$, a pronounced peak is observed at zero magnetic field which is attributed to the weak antilocalization effect. This feature originates from interference effects of holes in the presence of spin-orbit coupling. Indeed, for GeTe a bulk Rasbha effect in the valence band is theoretically predicted ${ }^{8,13}$. Furthermore, as discussed in more detail below, band bending at the surface in connection with according potential gradients may also result in spin-orbit coupling effects. The conductance traces shown in Fig. $2 \mathrm{~b}$ reveal a modulation pattern, which can be assigned to universal conductance fluctuations ${ }^{34}$. Their amplitude decreases with increasing the temperature mainly due to the reduction of the phase-coherence length ${ }^{35}$. From the autocorrelation function of the magnetoconductance, given by $F(\Delta B)=\langle G(B) G(B+\Delta B)\rangle$ $-\langle G(B)\rangle^{2}$, information on the phase-coherence length $l_{\phi}$ can be obtained $^{34}$. Here, $\langle\ldots\rangle$ is the average over the magnetic field. For quasi one-dimensional systems, i.e., systems with $l_{\phi}$ larger than the width of the wire, the correlation field $B_{\mathrm{c}}$ defined by $F\left(B_{\mathrm{c}}\right)=F$ $(0) / 2$, is inversely proportional to the phase-coherence length $l_{\phi}: B_{c} \sim \sqrt{3} \Phi_{0} /\left(\pi l_{\phi} d\right)$, with the magnetic flux quantum given by 

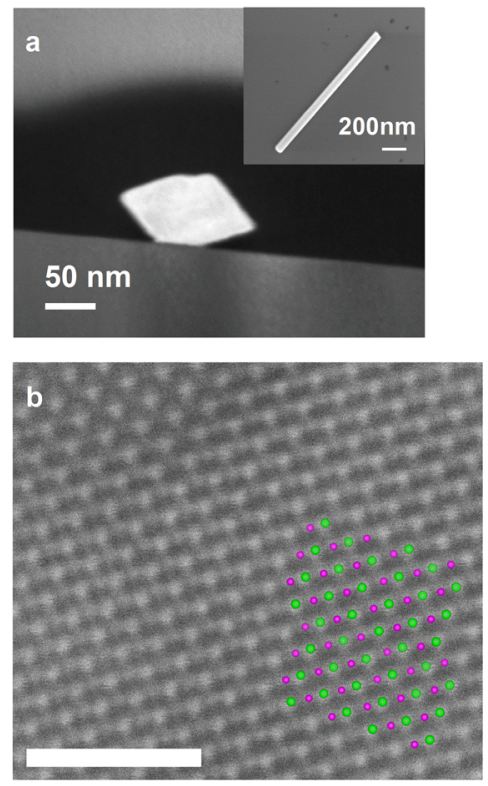

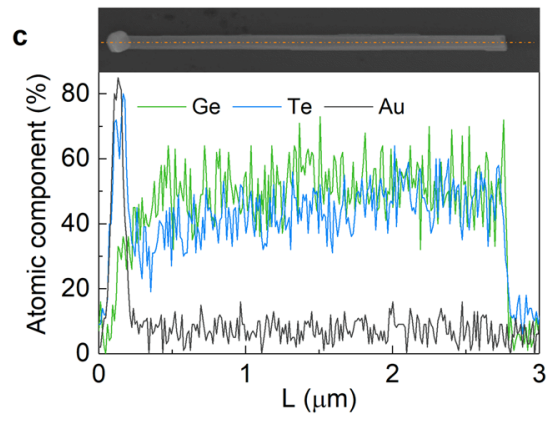

d

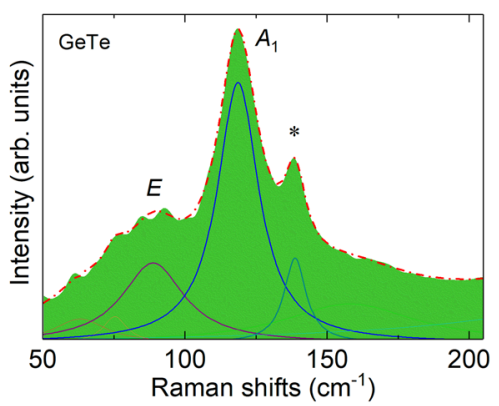

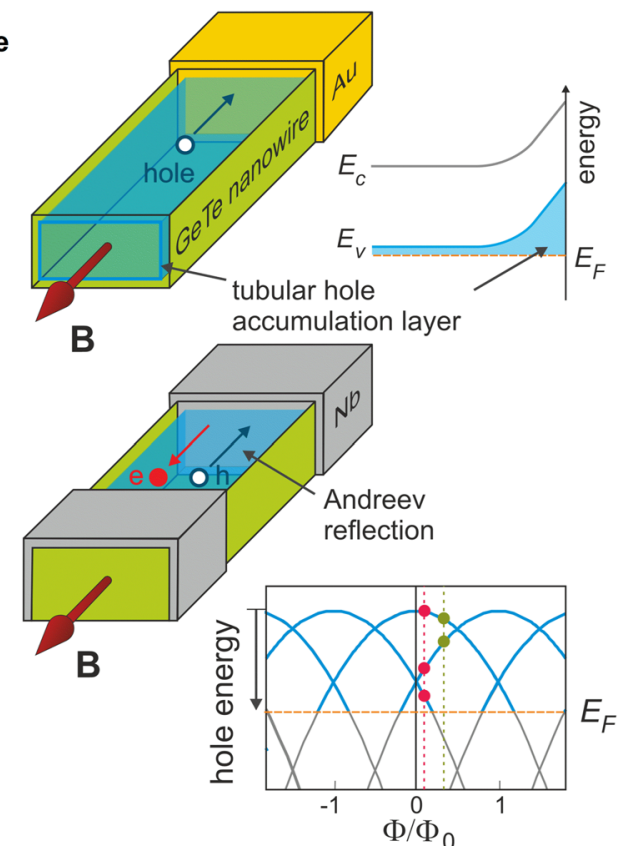

Fig. 1 Structural characterization and schematics of carrier transport. a Scanning transmission electron micrograph of GeTe NW in cross section. b HAADF image of GeTe NW cross section obtained via aberration corrected STEM with evident trigonal crystal structure, where light green atoms represent Te and magenta colored atoms indicate the position of Ge atoms. Scale bar: $2 \mathrm{~nm}$. c A SEM image of a GeTe nanowire and the corresponding atomic composition along the nanowire from Energy dispersive X-ray spectroscopy. $\mathbf{d}$ Experimental and fitted Raman spectra (solid lines) with mode assignments of a GeTe single nanowire. $E$ and $A_{1}$ are the first-order Raman-active modes while the feature $\left({ }^{\star}\right)$ arises from surface oxidation. e Top: Schematics of a GeTe nanowire with a tubular hole accumulation layer and a magnetic field along the wire axis. The scheme on the right shows the upwards bending of the bands at the surface, with $E_{\mathrm{F}}$ the Fermi energy as well as $E_{\mathrm{c}}$ and $E_{\mathrm{v}}$ the conduction band bottom and valence band top, respectively. Bottom: schematics of a GeTe nanowire contacted with Nb electrodes. At the interface Andreev reflection occurs. The schematics on the right shows the energy spectrum of hole states in a tubular conductor for different angular momenta as a function of the normalized flux $\Phi / \Phi_{0}$ for $E_{\mathrm{kin}}=0$. Note that for hole states the energy increases downwards and the effective mass is positive. The red and green dots indicate the occupied channels at different flux values.
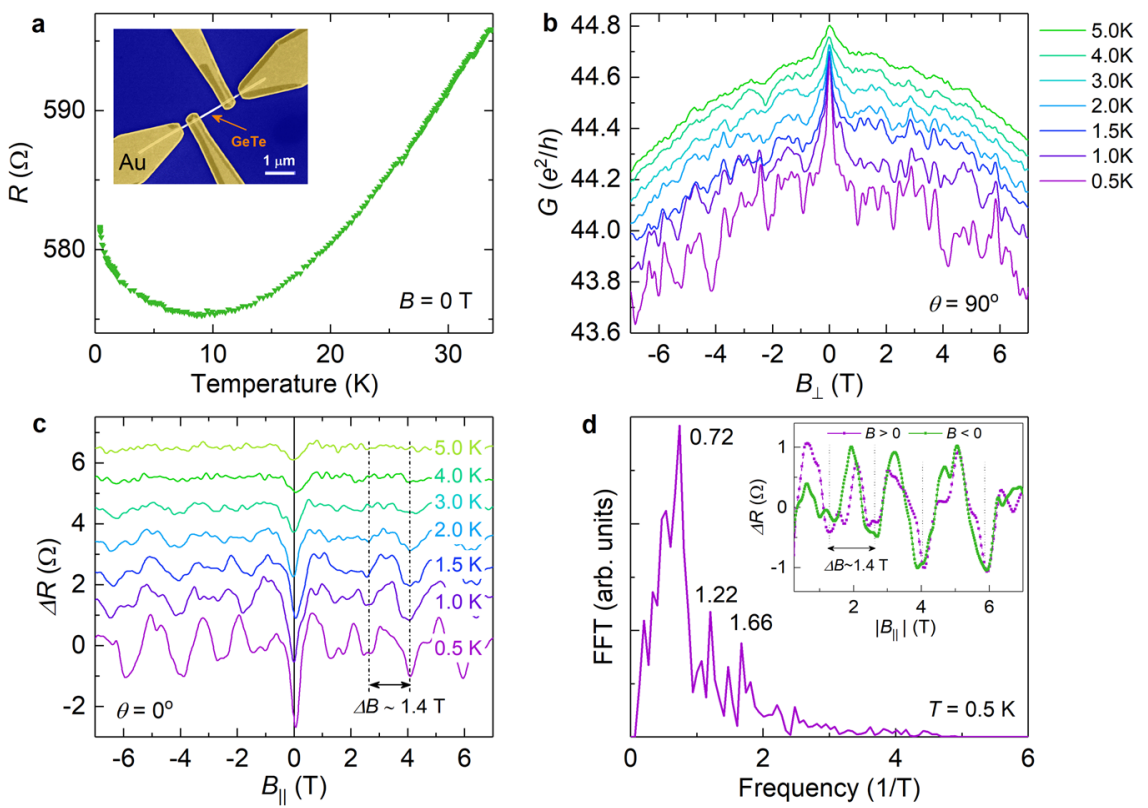

Fig. 2 Magnetotransport of a normal contacted GeTe nanowire. a Four-terminal resistance as a function of temperature at zero magnetic field of a $\mathrm{Au} / \mathrm{GeTe}$-nanowire/Au device. Inset: Scanning electron microscopy image of a GeTe NW contacted with Ti/Au leads. $\mathbf{b}$ Conductance in units of $e^{2} / h$ as a function of $B_{\perp}$ at temperatures from 0.5 to $5.0 \mathrm{~K}$. c Magnetoresistance oscillations after subtracting a parabolic background as a function of $B_{\|}$at temperatures from 0.5 to $5.0 \mathrm{~K}$. d Fast Fourier transform (FFT) of the magnetoresistance oscillations in a parallel magnetic field at $0.5 \mathrm{~K}$. The inset shows the corresponding resistance oscillations for the positive and negative magnetic field range. 
$\Phi_{0}=h / e$ (see also Supplementary Fig. 5 in Supplementary Note 2$)^{36,37}$. For the fluctuations measured at $0.5 \mathrm{~K}$, we extracted a correlation field of $B_{\mathrm{c}}=0.10 \mathrm{~T}$. Taking the nanowire width of $d$ $\sim 80 \mathrm{~nm}$ into account we obtain a phase-coherence length of about $280 \mathrm{~nm}$. The presence of conductance fluctuations and the fact that $l_{\phi}$ is considerably larger than the width of the nanowire confirms that the transport takes place in the diffusive quasi onedimensional regime.

Figure $2 \mathrm{c}$ shows the resistance $\Delta R$ after subtraction of a slowly varying background as a function of a magnetic field $B_{\|}$applied along the nanowire axis. For this magnetic field orientation one finds a regular oscillation pattern with an oscillation period of about $\Delta B=1.4 \mathrm{~T}$ (cf. Fig. $2 \mathrm{~d}$, inset). This is also confirmed by the fast Fourier spectrum of the measurement at $0.5 \mathrm{~K}$ depicted in Fig. $2 \mathrm{~d}$. Here, a pronounced peak is found at a frequency of $0.72 \mathrm{~T}^{-1}$, corresponding to the aforementioned period of $1.4 \mathrm{~T}$. In addition, some features at 1.22 and $1.66 \mathrm{~T}^{-1}$ are present.

We attribute the regular oscillations to Aharonov-Bohm type oscillations ${ }^{38}$ originating from transport in tubular hole states in a conductive surface accumulation layer penetrated by a magnetic flux $\Phi$ (cf. Fig. 1e, upper left schematics) ${ }^{35,39-42}$. The involvement of phase-coherent tubular states is supported by the large phasecoherence length being comparable to the circumference of the nanowire. We assume that in case of our GeTe nanowire, the holes accumulated in the surface channel are supplied from surface states. In fact, such a surface accumulation layer is mainly observed for narrow band gap materials, e.g., for InAs. Similar but inverted as in the case of InAs the GeTe electronic band structure exhibits some lower valence band maxima ${ }^{43,44}$, which may be responsible for the charge neutrality level of the surface states to be located within the bulk valence band. As a consequence, a tubular hole accumulation layer is formed at the surface ${ }^{45}$. Owing to the hole accumulation the valence band is bent upwards at the interface (cf. Fig. 1e and Supplementary Note 3 for more details on the formation of the accumulation layer).

In order to describe the transport through the tubular hole channel with a rectangular cross section in more detail, we assume a circular cross section ${ }^{42,46}$. In both cases quantized states will be formed, where the wave function along the circumference has to match after a full cycle. However, in case of a circular cross section the state components perpendicular to the nanowire axis can be described by coherent angular momentum states, which can be ordered according to their angular momentum quantum number $l$ of their angular momentum $L_{\mathrm{z}}$. This simplifies the theoretical description considerably. In the presence of a magnetic flux through the wire cross section the energy eigenstates are given by 39,41

$$
E=E_{\mathrm{kin}}+\frac{\hbar^{2}}{2 m^{*} r_{0}^{2}}\left(l-\frac{\Phi}{\Phi_{0}}\right)^{2}
$$

with $E_{\text {kin }}$ the kinetic energy along the wire, $m^{*}$ the effective hole mass, and $r_{0}$ the radius of the tubular channel. The energy is periodic in $\Phi_{0}=h / e$.

A schematics of the energy spectrum is given in Fig. 1e, bottom. In a ballistic picture the conductance is determined by the number of occupied hole channels above the Fermi level $E_{\mathrm{F}}$, e.g., three channels for a small flux (red dots in the schematics) and two channels for a slightly larger flux (green dots) ${ }^{39}$. Thus, when the magnetic field is increased, the number of occupied hole channels changes periodically with the period of $\Phi_{0}$. In the nonballistic diffusive case, a similar flux-periodic modulation is expected as well ${ }^{47}$. However, it must be stressed that the actual presence of the Aharonov-Bohm oscillations does not depend on the hole concentration in the surface gas.
From the measured period of $\Delta B=1.4 \mathrm{~T}$ of our nanowire we can deduce the expected area $S$ encircled by the surface channel from $\Phi_{0}=S \times \Delta B$, which results in a value of about $S=3000$ $\mathrm{nm}^{2}$, corresponding to a diameter of approximately $60 \mathrm{~nm}$ for a circular cross section. For our nanowire with a width of $80 \mathrm{~nm}$, we estimate an area of about $5000 \mathrm{~nm}^{2}$ assuming a circular cross section. Obviously, the expected cross section is smaller than the actual geometrical cross sectional area of our GeTe nanowire. The discrepancy may be attributed to the fact that the nanowire surface is oxidized so that the hole accumulation layer is slightly pushed inside. Furthermore, the radial component wave function usually has an extension of a few nanometers, which also leads to a reduction of the effective cross sectional area. As mentioned above, some weaker maxima at higher frequencies, i.e., at 1.22 and $1.66 \mathrm{~T}^{-1}$, are found in the Fourier spectrum. These features may be related to higher harmonics or to Altshuler-Aronov-Spivak oscillations comprising a $h / 2 e$ periodicity ${ }^{48}$.

We now turn to measurements of GeTe nanowires equipped with superconducting contacts. The inset of Fig. 3a shows a GeTe $\mathrm{NW}$-based junction device with $\mathrm{Nb}$ contacts. The nanowire has a width of $80 \mathrm{~nm}$, while the width and separation of $\mathrm{Nb}$ leads are about 500 and $90 \mathrm{~nm}$, respectively. The outer $\mathrm{Nb}$ leads are employed to apply bias current, while the inner pair of contacts serve as voltage probes ${ }^{49,50}$. Figure 3 a shows the resistance $R$ at zero magnetic field as a function of temperature. The measurement shown in Fig. $3 \mathrm{a}$ indicates that the $\mathrm{Nb}$ electrodes have a transition temperature of about $T_{\mathrm{c}, \mathrm{Nb}}=7.0 \mathrm{~K}$. From the relation of the $T_{\mathrm{c}, \mathrm{Nb}} \approx 3.9$, with $k_{\mathrm{B}}$ the Boltzmann constant and critical temperature $T_{c, N b}$, we estimate the $\mathrm{Nb}$ energy gap $\Delta_{0}$ to be about $1.2 \mathrm{meV}$ at zero temperature ${ }^{51,52}$. Moreover, there is another drop in the temperature region between 1.0 and $2.0 \mathrm{~K}$, which is assigned to the superconducting transition temperature $T_{c, N W}$ of the $\mathrm{Nb} / \mathrm{GeTe}-\mathrm{NW} / \mathrm{Nb}$ junction. The two-step feature has been observed before in Josephson junctions with a normal metal ${ }^{53}$ and a semiconductor weak $\operatorname{link}^{54}$ as well as in topological insulator-based junctions ${ }^{55}$. The normal state four-terminal resistance $\left(R_{\mathrm{N}}\right)$ of the present $\mathrm{Nb} / \mathrm{GeTe}-\mathrm{NW} / \mathrm{Nb}$ junction is about $355 \Omega$ at temperatures above $T_{\mathrm{c}, \mathrm{Nb}}$.

Figure $3 \mathrm{~b}$ shows the current-voltage $(I-V)$ characteristics and corresponding differential resistance $(\mathrm{d} V / \mathrm{d} I)$ curve measured at $0.4 \mathrm{~K}$. At low bias currents a superconducting state is observed with a critical current of $200 \mathrm{nA}$. In the $\mathrm{d} V / \mathrm{d} I$ trace modulations are observed, which we assign to subharmonic gap structure caused by multiple Andreev reflections, as described within the Octavio-Tinkham-Blonder-Klapwijk (OTBK) model ${ }^{56,57}$. As indicated in Fig. $3 \mathrm{~b}$, peaks corresponding to $2 \Delta_{0} / 3$ and $\Delta_{0}$ are present, while in the region around $2 \Delta_{0}$ one only finds a broad indentation. Although relatively weak, the observed subharmonic gap features basically follow the expected modulations predicted by the OTBK model. Several reasons can be made out to be responsible for the dampening of the subharmonic gap structures. First of all, the finite junction length in relation to the superconducting coherence length can cause a weakening of the subharmonic gap structures ${ }^{58}$. Furthermore, in our structure the interface between the $\mathrm{Nb}$ and GeTe is laterally not sharply defined, i.e., the effective junction length might vary. This probably also leads to a broadening of the subharmonic gap structures. In addition to the regular subharmonic gap structure we also found sharp peak indicated by (1) in Fig. 3b. We attribute this feature to a parasitic superconducting switching event, probably due to some defect in the superconducting electrodes in series.

As a further step, the temperature dependence of the $I-V$ characteristics is investigated (cf. Fig. 3c). Obviously, the superconducting range at small bias currents (indicated by a rectangle) shrinks and finally vanishes upon increasing the temperature 

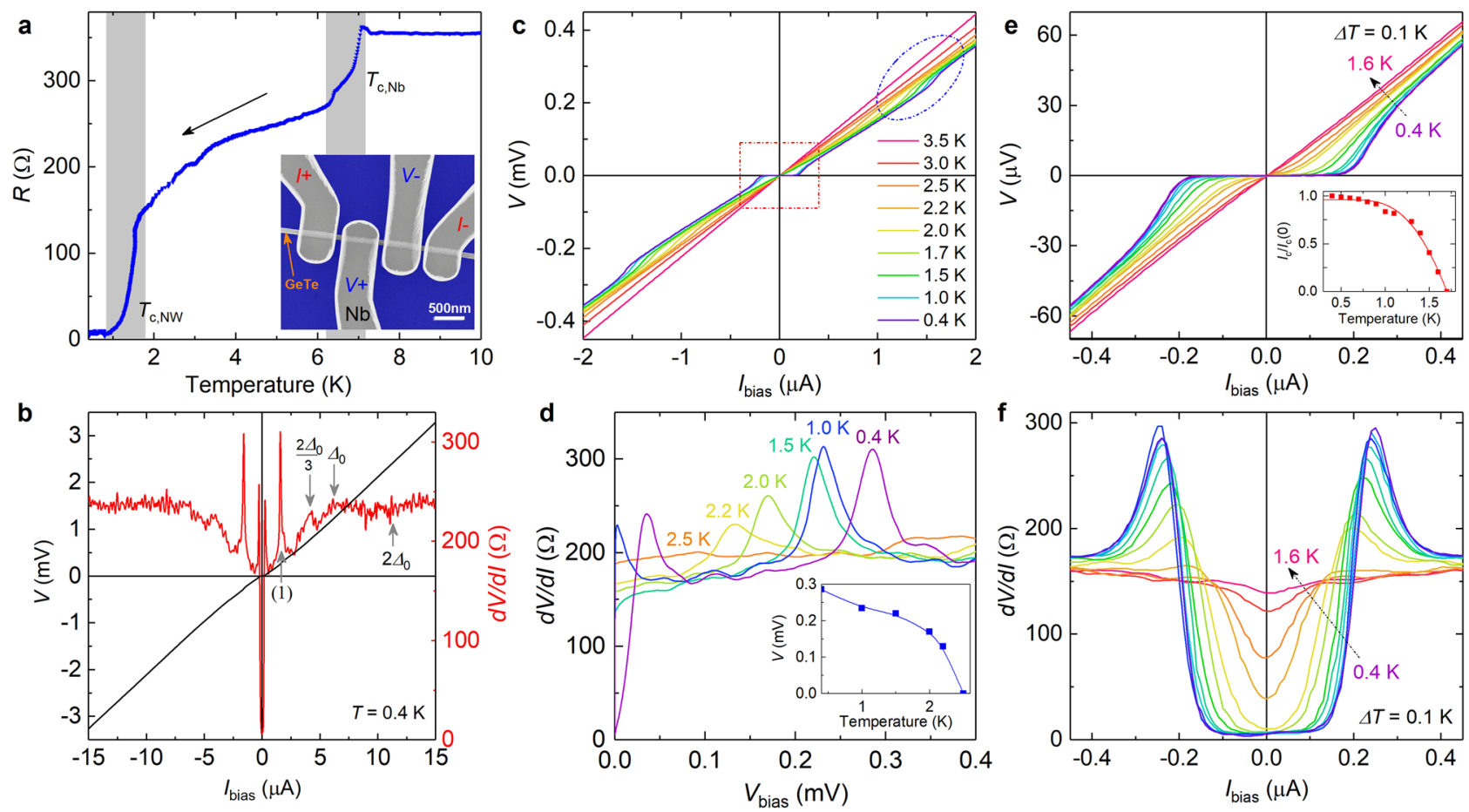

Fig. 3 Transport characteristic of $\mathbf{N b} / \mathbf{G e T e}$-nanowire/Nb junction at zero magnetic field. a Resistance $R$ of a $\mathrm{Nb} / \mathrm{GeTe}-\mathrm{nanowire} / \mathrm{Nb}$ junction during a cooling process. There are two transitions $\left(T_{c, N b}\right.$ and $\left.T_{c, N W}\right)$ labeled by vertical shadows. Inset: A scanning electron microscopy image of a $\mathrm{Nb} / \mathrm{GeTe}-$ nanowire/Nb junction. $\mathbf{b} I-V$ characteristics and corresponding $\mathrm{d} V / \mathrm{d} /$ trace of a GeTe-based junction in a high bias current range at $0.4 \mathrm{~K}$. The subgap features at $2 \Delta_{0} / 3$ and $\Delta_{0}$ as well as the location of $2 \Delta_{0}$ are indicated by arrows. Furthermore, an additional sharp feature is found at (1). $\mathbf{c} I-V$ curves as a function of large bias current range. The superconducting range at small bias currents is indicated by an rectangle and the step feature at around $\pm 1.5 \mu \mathrm{A}$ can be found within the ellipse. $\mathbf{d}$ Corresponding $\mathrm{dV} / \mathrm{d} /$ traces as a function of bias voltage at various temperatures. Inset: Position as a function of temperature of the peak assigned feature (1) indicated in $\mathbf{b}$. e $l-V$ characteristics and $\mathbf{f}$ the corresponding $d V / d l$ curves in a small bias current range at temperatures between 0.4 and $1.6 \mathrm{~K}$. The inset of $\mathbf{e}$ is the experimental (dots) and fit (solid line) $I_{d} / I_{c}(0)$ as a function of temperature.

from 0.4 to $3.5 \mathrm{~K}$. The same is true for the step feature at around $\pm 1.5 \mu \mathrm{A}$. Regarding the latter, the corresponding $d V / \mathrm{d} I$ traces as a function of bias voltage are shown in Fig. 3d for temperatures in the range from 0.4 to $2.5 \mathrm{~K}$. One finds that the feature corresponding to peak (1) in Fig. 3b shifts towards smaller bias voltages and eventually vanishes at a temperature of $2.5 \mathrm{~K}$ ( $\mathrm{cf}$. Fig. 3d, inset). The strong temperature dependence confirms that this feature is directly related to the superconducting state.

Figure 3 e shows the $I-V$ characteristics of the GeTe-NW based Josephson junction in a small bias current range at temperatures ranging from 0.4 to $1.6 \mathrm{~K}$. At $0.4 \mathrm{~K}$ the critical current $I_{\mathrm{c}, \mathrm{NW}}$ is about $200 \mathrm{nA}$. As the temperature increases, the superconducting plateau diminishes and disappears at about $1.6 \mathrm{~K}$. The according corresponding differential resistance traces are shown in Fig. 3f. The dependence of normalized critical current $I_{c} / I_{c}(0)$ as a function of temperature are depicted in the inset of Fig. 3e. The temperature dependence of the supercurrent can be assigned to a metallic diffusive junction ${ }^{59}$. However, since no reliable data on the diffusion constant and Thouless energy can be obtained, we have refrained from a detailed analysis.

$\mathrm{The} \mathrm{Nb} / \mathrm{GeTe}-\mathrm{NW} / \mathrm{Nb}$ junctions were measured in a magnetic field in two orientations, i.e., parallel to the nanowire axis and perpendicular to the substrate plane. For the perpendicular configuration the critical field $B_{c, \perp}$ was determined to be around $4 \mathrm{~T}$ while for the parallel case we got $B_{\mathrm{c}, \|} \approx 5 \mathrm{~T}$. (In Supplementary Note 4 measurements at various tilt angles of the magnetic field are shown.) Figure $4 \mathrm{a}, \mathrm{b}$ depict the evolution of $I-V$ and corresponding $\mathrm{d} V / \mathrm{d} I$ characteristics of the $\mathrm{Nb} / \mathrm{GeTe}-\mathrm{NW} / \mathrm{Nb}$ junction in a perpendicular magnetic field $\left(B_{\perp}\right)$ at $0.5 \mathrm{~K}$. With increasing magnetic field, the supercurrent is suppressed and then disappears, as can be seen in more detail in Fig. 4a (inset). The features at around \pm 1.6 and $\pm 4 \mu \mathrm{A}$ found in the $\mathrm{d} V / \mathrm{d} I$ trace at zero field shift towards zero bias upon increasing the magnetic field. The shift can be attributed to the reduced superconducting gap when a magnetic field is applied. As shown in Fig. 4c, we find a monotonous decrease of the critical current $I_{c, \perp}$ with increasing $B_{\perp}$. A complete suppression of $I_{c, \perp}$ occurs at around $4 \mathrm{~T}$. A similar monotonous decrease of critical current with $B_{\perp}$ was observed in planar $\mathrm{Nb} / \mathrm{Au} / \mathrm{Nb}^{53}$ as well as in semiconductor nanowire based Josephson junctions ${ }^{52,60}$. It can be explained within the framework of a theoretical model for the proximity effect in diffusive narrow-width Josephson junctions ${ }^{61}$. Within that model the decay of $I_{c, \perp}$ can be described by ${ }^{53,61}$ : $I_{c, \perp}(B)=I_{c, \perp}(0) \cdot a e^{-b\left(B S / \Phi_{0}\right)^{2}}$. Here, $I_{c, \perp}(0)$ is the critical current at $B=0 \mathrm{~T}$. The $a$ and $b$ are fit parameters and $S$ is the effective area $(80 \mathrm{~nm} \times 90 \mathrm{~nm})$ of the $\mathrm{Nb} / \mathrm{GeTe} / \mathrm{Nb}$ junction perpendicular to magnetic field. In the model we do not distinguish between bulk and surface contributions. The fit curve from theory (solid line) agrees well with the experimental data for fit parameters $a$ and $b$ of 1.04 and 0.27 , respectively.

In the case that the applied magnetic field is parallel to the GeTe wire axis $\left(B_{\|}\right)$, the supercurrent is modulated when the magnetic field is increased, as can be seen in Fig. 4d, e. Furthermore, similar to the previous case where $B_{\perp}$ was applied the peak features in the $\mathrm{d} V / \mathrm{d} I$ traces shift towards zero bias upon increasing of $B_{\|}$. In Fig. 4e the ranges where a Josephson supercurrent is present is indicated. The corresponding values of $I_{c, \|}$ as a function of $B_{\|}$are plotted in Fig. $4 \mathrm{f}$. One clearly finds an oscillatory behavior of $I_{\mathrm{c}, \|}$. The oscillation period of $\Delta B_{\|}$is very similar to the period of the sample with normal contacts, as 

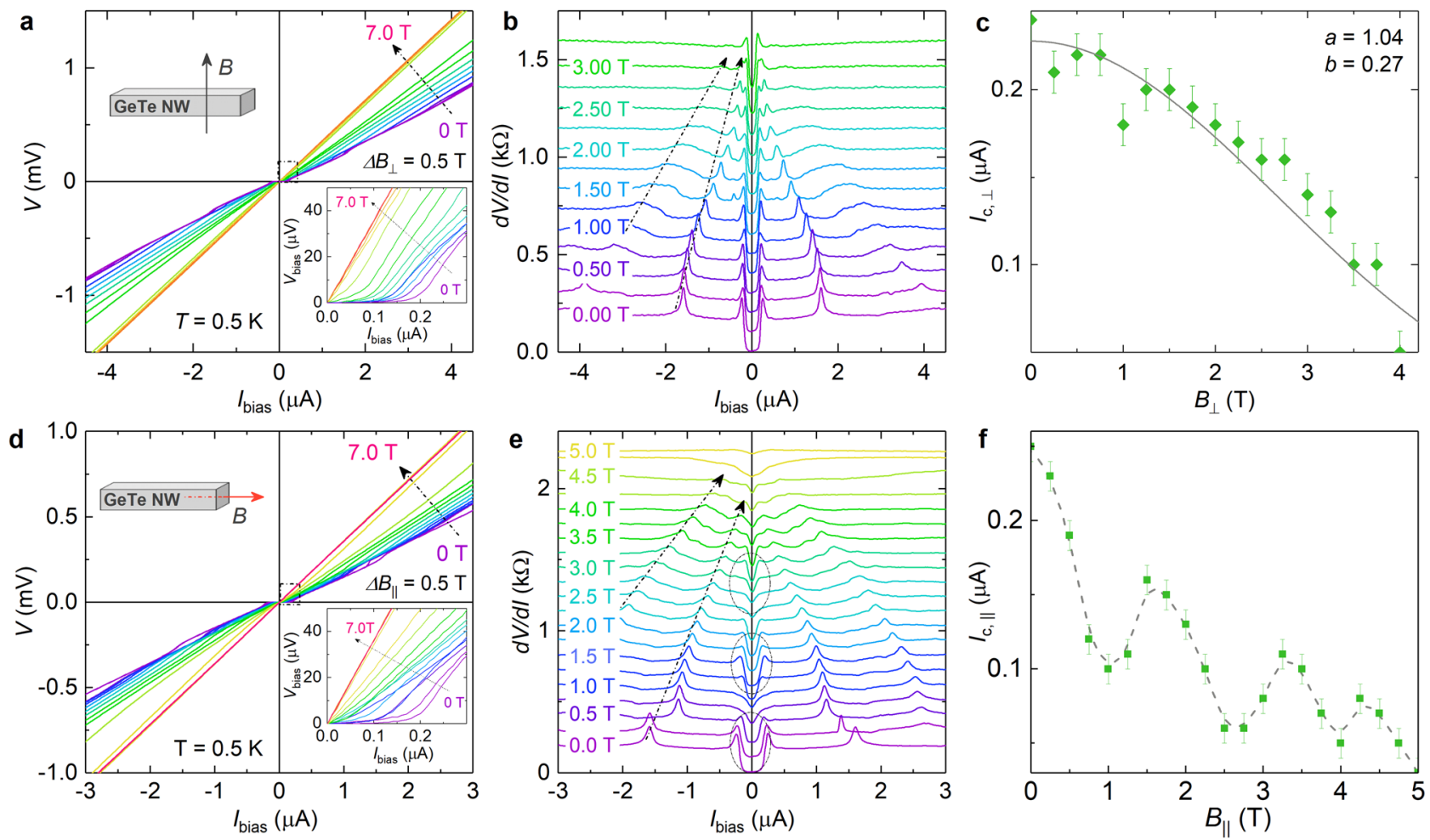

Fig. 4 Magnetotransport transport in Nb/GeTe-nanowire/Nb junction. a $/-V$ characteristics and $\mathbf{b}$ the corresponding $\mathrm{dV} / \mathrm{d} / \mathrm{traces}$ of a $\mathrm{Nb} / \mathrm{GeTe}-\mathrm{NW} / \mathrm{Nb}$ junction as a function of bias current at $0.5 \mathrm{~K}$ by applying various perpendicular magnetic fields $B_{\perp}$. The insets in a show the orientation of the magnetic field and a detail at small bias currents. c Experimental (dots) and fitted (solid line) critical current $I_{c, \perp}$ as a function of magnetic field ( $B_{\perp}$ ) based on the $\mathrm{d} V / \mathrm{d} /$ curves at a small bias current region (cf. inset in $\mathbf{a}$ ). The error bars represent the resolution of the current bias. $\mathbf{d} I-V$ characteristics and $\mathbf{e}$ the corresponding $\mathrm{dV} / \mathrm{d} /$ traces as a function of bias current at $0.5 \mathrm{~K}$ by applying various magnetic field parallel to the GeTe NW axis ( $\left.B_{\|}\right)$. The insets in $\mathbf{d}$ show a details of the characteristics around zero current bias and illustrate the magnetic field orientation with respect to the nanowire. All $\mathrm{dV} / \mathrm{d} / \mathrm{curves}$ in $\mathbf{b}$ and e are shifted vertically for clarity. In e the ranges where a supercurrent is observed are indicated by the ellipses. $\mathbf{f}$ Critical current $I_{c, l l}$ as a function of magnetic field based on the $\mathrm{dV} / \mathrm{dl}$. The dashed line is a guide to the eye.

discussed before. Since the cross section of the nanowire is very similar to the previous one, we can deduce that oscillations of $I_{c, \|}$ are periodic with a single magnetic flux quantum $\Phi_{0}=h / e$. We interpret the behavior by a Josephson supercurrent which is carried by coherent closed-loop states in the surface accumulation layer at the GeTe nanowire surface. As illustrated in Fig. 1e, bottom, the supercurrent originates from phase-coherent Andreev retro-reflections of tubular hole and electron states at both $\mathrm{GeTe} / \mathrm{Nb}$ interfaces ${ }^{62}$. In each cycle a Cooper pair is effectively transported from one $\mathrm{Nb}$ electrode to the other one. In the measurements of GeTe nanowire with normal contacts, we found that the resistance was modulated with a magnetic flux quantum because of the periodic change of the number of occupied transport channels. In case of superconducting electrodes, the magnitude of the Josephson supercurrent is also considered to be determined by the number of hole and electron transport channels, hence a flux periodic modulation is expected as well. As a matter of fact, a magnetic flux modulation was found before in junctions based on GaAs/InAs core/shell nanowires ${ }^{41,63}$. However, in that case no clear supercurrent was observed and a period of $\Phi_{0} / 2$ was found.

In summary, magnetotransport of GeTe nanowire-based devices have been investigated in a four-terminal configuration. The temperature dependent resistance of the normal metal contacted $\mathrm{Au} / \mathrm{GeTe}$-nanowire/Au devices reveals that $\mathrm{GeTe}$ exhibits a semiconducting behavior until $0.4 \mathrm{~K}$. At low temperatures universal conductance fluctuations were observed which allowed us to extract the phase-coherence length $l_{\phi}$ being as large as $280 \mathrm{~nm}$ at $0.5 \mathrm{~K}$. The weak antilocalization feature observed around zero magnetic field indicates the presence of spin-orbit coupling in the valence band. Even more, when a parallel magnetic field is applied regular Aharonov-Bohm type oscillations are found, which are attributed to the formation of a hole accumulation layer at the nanowire surface. In the case of $\mathrm{Nb} / \mathrm{GeTe}-\mathrm{NW} / \mathrm{Nb}$ Josephson junctions, we observed a critical supercurrent of about $200 \mathrm{nA}$ at $0.4 \mathrm{~K}$. The temperature dependence of $T_{c, N W}$ can be explained in the framework of a diffusive junction. With increasing a perpendicular magnetic field the critical current decreases monotonously. This feature can be explained within theoretical models covering the small junction limit. For magnetic fields applied parallel to the nanowire axis regular oscillations of the critical current are observed, which are attributed to a supercurrent carried by the surface accumulation layer in the GeTe nanowires. For future studies it would be interesting to find out if the transport phenomena based on a surface accumulation channel can also be observed in GeTe nanostructures with different geometries, e.g., zigzag nanowires or nanohelices ${ }^{22,25}$.

Our investigations showed that distinct phase-coherent phenomena can be observed in GeTe nanowire structures. The very pronounced and sharp weak antilocalization feature indicates that the corresponding spin precession length is very short, which implies that spin manipulation might be possible on a very small length scale ${ }^{8}$. So far, we could not discriminate between Rashba spin-orbit coupling originating from the potential gradient connected to the surface hole accumulation layer and the electric field due to the polarization in the bulk. In order to gain more insight in the contributions of the surface and the giant bulk Rashba effect, it would be very interesting to perform measurements on 
gated nanowires. This is also of interest with respect to the switching effect based on the ferroelectric properties of GeTe. All in all, the presence of spin-orbit coupling in combination with the superconducting proximity effect make these nanowires very attractive for applications in the field of topological quantum computation. In this context, it is also worth-mentioning that by combining GeTe with three-dimensional topological insulators in a superlattice the topological properties can be tailored ${ }^{21}$.

\section{Methods}

GeTe nanowire synthesis. The synthesis of GeTe nanowires are achieved by a Aucatalyzed vapor-liquid-solid growth. Firstly, the $\mathrm{Si}(100)$ substrates with a native oxide were cleaned with acetone, iso-propyl alcohol, and deionized water in an ultrasonic cleaning bath, and then treated in a piranha solution to remove organic residues. After that, the substrates were immersed in Au nanoparticles solution for a few min and rinsed with deionized water. Finally, bulk GeTe $(99.99 \%$, SigmaAldrich) was evaporated at the center of a horizontal tube furnace, and the reaction product was collected downstream on a $\mathrm{Si} / \mathrm{SiO}_{2}$ substrate covered with colloidal $\mathrm{Au}$ nanoparticles. Specifically, the furnace was evacuated and purged three times with Ar gas. Then it was heated to $400{ }^{\circ} \mathrm{C}$ and persisted for $8 \mathrm{~h}$. During this program, the Ar flow rate and the pressure in the quartz tube were about $140 \mathrm{sccm}$ and 10 Torr, respectively.

GeTe-based device fabrication. In order to contact a single GeTe nanowire with $\mathrm{Nb}$ or $\mathrm{Au}$ electrodes, the as-grown GeTe NWs were transferred to a $\mathrm{Si} / \mathrm{SiO}_{2} / \mathrm{Si}_{3} \mathrm{~N}_{4}$ substrate with predefined markers for electron beam lithography. A two-layer (copolymer/950 K) polymethyl methacrylate (PMMA) resist system was adopted to realized an ideal shape of $\mathrm{Nb}$ or Au leads. In order to obtain a transparent interface between electrodes and $\mathrm{GeTe}$, the samples were exposed to an oxygen plasma to remove resist residues on the contact area. Before the deposition of the electrodes on a single nanowire in a magnetron sputter chamber with a DC power of $250 \mathrm{~W}$, the samples were lightly cleaned by $45 \mathrm{~s} \mathrm{Ar}^{+}$plasma milling to remove native oxidation layers on the GeTe surface.

Morphology and composition characterizations. The morphology and chemical composition of the GeTe nanowires was determined by SEM and EDX, respectively. In addition, a cross sectional specimen has been prepared by focused ion beam techniques. High-angle annular dark field imaging in the STEM has been employed to investigate the cross sectional shape of the nanowires. In addition, the crystalline structure has been investigated by $\mathrm{x}$-ray diffraction and Raman scattering. (In Supplementary Note 1 additional information on the sample characterization is provided.)

Magnetotransport measurements. The temperature and magnetic field dependent $I-V$ characteristics and $\mathrm{d} V / \mathrm{d} I$ curves were measured using a standard lockin technology in a ${ }^{3} \mathrm{He}$ cryostat with a base temperature of $0.4 \mathrm{~K}$ and a magnetic field range from -8 to $8 \mathrm{~T}$. A four-terminal current driven geometry is employed to directly measuring the voltage drop across the inner section of GeTe nanowire between the nearest internal electrodes. The external pair of electrodes was used for current bias ${ }^{55}$. The differential resistance $(\mathrm{d} V / \mathrm{d} I)$ is gained by superimposing a small AC current of $10 \mathrm{nA}$ at $9.4 \mathrm{~Hz}$ on a DC bias current. Note that the applied bias current should be as small as possible to avoid electron heating and damage of the GeTe-based nanodevices.

\section{Data availability}

The data that support the findings of this study is available from the authors on a reasonable request. The source data underlying Figs. 1-4 and Supplementary Figs. 3, 6, and 8 are provided as a Source Data file from the Jülich DATA repository (https://doi. org/10.26165/JUELICH-DATA/M1IQVG).

Received: 15 September 2020; Accepted: 5 January 2021; Published online: 02 February 2021

\section{References}

1. Wuttig, M. \& Yamada, N. Phase-change materials for rewriteable data storage. Nat. Mater. 6, 824-832 (2007).

2. Lencer, D. et al. A map for phase-change materials. Nat. Mater. 7, 972-977 (2008).

3. Guo, S. et al. Intrinsic evolutions of dielectric function and electronic transition in tungsten doping $\mathrm{Ge}_{2} \mathrm{Sb}_{2} \mathrm{Te}_{5}$ phase change films discovered by ellipsometry at elevated temperatures. Appl. Phys. Lett. 106, 052105 (2015).
4. Hong, M., Zou, J. \& Chen, Z.-G. Thermoelectric GeTe with diverse degrees of freedom having secured superhigh performance. Adv. Mater. 31, 1807071 (2019).

5. Perumal, S., Roychowdhury, S. \& Biswas, K. High performance thermoelectric materials and devices based on GeTe. J. Mater. Chem. C 4, 7520-7536 (2016).

6. Zhu, M. et al. Unique bond breaking in crystalline phase change materials and the quest for metavalent bonding. Adv. Mater. 30, 1706735 (2018).

7. Park, J.-W. et al. Optical properties of $\left(\mathrm{GeTe}, \mathrm{Sb}_{2} \mathrm{Te}_{3}\right)$ pseudobinary thin films studied with spectroscopic ellipsometry. Appl. Phys. Lett. 93, 021914 (2008).

8. Di Sante, D., Barone, P., Bertacco, R. \& Picozzi, S. Electric control of the giant Rashba effect in bulk GeTe. Adv. Mater. 25, 509-513 (2013).

9. Singh, D. J. Optical properties of cubic and rhombohedral GeTe. J. Appl. Phys. 113, 203101 (2013)

10. Picozzi, S. Ferroelectric Rashba semiconductors as a novel class of multifunctional materials. Front. Phys. 2, 10 (2014).

11. Bahramy, M. S. \& Ogawa, N. Bulk Rashba semiconductors and related quantum phenomena. Adv. Mater. 29, 1605911 (2017).

12. Boschker, J. E., Wang, R. \& Calarco, R. GeTe: a simple compound blessed with a plethora of properties. CrystEngComm 19, 5324-5335 (2017).

13. Liebmann, M. et al. Giant Rashba-type spin splitting in ferroelectric GeTe (111). Adv. Mater. 28, 560-565 (2016).

14. Krempaský, J. et al. Disentangling bulk and surface Rashba effects in ferroelectric $\alpha$-GeTe. Phys. Rev. B 94, 205111 (2016).

15. Elmers, H. J. et al. Spin mapping of surface and bulk Rashba states in ferroelectric $\alpha$-GeTe(111) films. Phys. Rev. B 94, 201403 (2016).

16. Krempaský, J. et al. Fully spin-polarized bulk states in ferroelectric GeTe. Phys. Rev. Res. 2, 013107 (2020).

17. Hein, R. A., Gibson, J. W., Mazelsky, R., Miller, R. C. \& Hulm, J. K. Superconductivity in germanium telluride. Phys. Rev. Lett. 12, 320-322 (1964).

18. Smith, J. L. \& Stiles, P. Superconducting energy gap in semiconducting GeTe. J. Low Temp. Phys. 26, 101-110 (1977).

19. Narayan, V., Nguyen, T.-A., Mansell, R., Ritchie, D. \& Mussler, G. Interplay of spin-orbit coupling and superconducting correlations in germanium telluride thin films. Phy. Status Solidi R 10, 253-259 (2016).

20. Narayan, V. et al. Long-lived nonequilibrium superconductivity in a noncentrosymmetric Rashba semiconductor. Phys. Rev. B 100, 024504 (2019).

21. Tominaga, J., Kolobov, A. V., Fons, P., Nakano, T. \& Murakami, S. Ferroelectric order control of the Dirac-semimetal phase in $\mathrm{GeTe}-\mathrm{Sb}_{2} \mathrm{Te}_{3}$ superlattices. Adv. Mater. Interfaces 1, 1300027 (2014)

22. Meister, S. et al. Synthesis and characterization of phase-change nanowires. Nano Lett. 6, 1514-1517 (2006).

23. Meister, S., Schoen, D. T., Topinka, M. A., Minor, A. M. \& Cui, Y. Void formation induced electrical switching in phase-change nanowires. Nano Lett. 8, 4562-4567 (2008).

24. Longo, M. et al. Au-catalyzed self assembly of GeTe nanowires by MOCVD. J. Cryst. Growth 315, 152-156 (2011).

25. Yu, D., Wu, J., Gu, Q. \& Park, H. Germanium telluride nanowires and nanohelices with memory-switching behavior. J. Am. Chem. Soc. 128, 8148-8149 (2006).

26. Bragaglia, V. et al. Far-infrared and Raman spectroscopy investigation of phonon modes in amorphous and crystalline epitaxial $\mathrm{GeTe}-\mathrm{Sb}_{2} \mathrm{Te}_{3}$ alloys. Sci. Rep. 6, 28560 (2016).

27. Shaltaf, R., Gonze, X., Cardona, M., Kremer, R. K. \& Siegle, G. Lattice dynamics and specific heat of $\alpha$-GeTe: Theoretical and experimental study. Phys. Rev. B 79, 075204 (2009).

28. Andrikopoulos, K. S. et al. Raman scattering study of the $\alpha$-GeTe structure and possible mechanism for the amorphous to crystal transition. J. Phys. Condens. Matter 18, 965-979 (2006).

29. Pawbake, A. et al. Pressure-induced phase transitions in germanium telluride: Raman signatures of anharmonicity and oxidation. Phys. Rev. Lett. 122, 145701 (2019)

30. Shalini, A. et al. Coherent phonon modes of crystalline and amorphous $\mathrm{Ge}_{2} \mathrm{Sb}_{2} \mathrm{Te}_{5}$ thin films: a fingerprint of structure and bonding. J. Appl. Phys. 117, 025306 (2015).

31. Weyrich, C. et al. Growth, characterization, and transport properties of ternary $\left(\mathrm{Bi}_{1-x} \mathrm{Sb}_{x}\right)_{2} \mathrm{Te}_{3}$ topological insulator layers. J. Phys. Condens. Matter 28, 495501 (2016)

32. Lee, P. A. \& Ramakrishnan, T. V. Disordered electronic systems. Rev. Mod. Phys. 57, 287-337 (1985).

33. Bergmann, G. Weak localization in thin films, a time-of-flight experiment with conduction electrons. Phys. Rep. 107, 1-58 (1984).

34. Lee, P. A. \& Stone, A. D. Universal conductance fluctuations in metals. Phys Rev. Lett. 55, 1622-1625 (1985).

35. Arango, Y. C. et al. Quantum transport and nano angle-resolved photoemission spectroscopy on the topological surface states of single $\mathrm{Sb}_{2} \mathrm{Te}_{3}$ nanowires. Sci. Rep. 6, 29493 (2016)

36. Ferry, D. K., Goodnick, S. \& Bird, S. M. Transport in Nanostructures. (Cambridge University Press, Cambridge, 2009). 
37. Beenakker, C. W. J. \& van Houten, H. in (eds Ehrenreich, H. \& Turnbull, D.) Solid State Physics, vol. 44, 1-228 (Academic, New York, 1991).

38. Aharonov, Y. \& Bohm, D. Significance of electromagnetic potentials in the quantum theory. Phys. Rev. 115, 485-491 (1959).

39. Richter, T. et al. Flux quantization effects in InN nanowires. Nano Lett. 8 , 2834-2838 (2008).

40. Peng, $\mathrm{H}$. et al. Aharonov-Bohm interference in topological insulator nanoribbons. Nat. Mater. 9, 225-229 (2010).

41. Gül, O. et al. Flux periodic magnetoconductance oscillations in GaAs/InAs core/shell nanowires. Phys. Rev. B 89, 045417 (2014).

42. Cho, S. et al. Aharonov-Bohm oscillations in a quasi-ballistic threedimensional topological insulator nanowire. Nat. Commun. https://doi.org/ 10.1038/ncomms8634 (2015)

43. Polatoglou, H. M., Theodorou, G. \& Economou, N. A. Physics of Narrow Gap Semiconductors (eds Gornik, E. et al.) 221-225 (Springer, Berlin, Heidelberg, 1982).

44. Jain, A. et al. The materials project: a materials genome approach to accelerating materials innovation. APL Mater. 1, 011002 (2013).

45. Lüth, H. Solid Surfaces, Interfaces and Thin Films. 6th edn (Springer, Berlin, Heidelberg, 2015).

46. Ziegler, J. et al. Probing spin helical surface states in topological $\mathrm{HgTe}$ nanowires. Phys. Rev. B 97, 035157 (2018).

47. Rosdahl, T. O., Manolescu, A. \& Gudmundsson, V. Spin and impurity effects on flux-periodic oscillations in core-shell nanowires. Phys. Rev. B 90, 035421 (2014).

48. Al'tshuler, B. L., Aronov, A. G. \& Spivak, B. Z. Aharonov-bohm effect in disordered conductors. JETP Lett. 33, 101-103 (1981).

49. Courtois, H., Gandit, P. \& Pannetier, B. Proximity-induced superconductivity in a narrow metallic wire. Phys. Rev. B 52, 1162-1166 (1995).

50. Skryabina, O. V. et al. Josephson coupling across a long single-crystalline $\mathrm{Cu}$ nanowire. Appl. Phys. Lett. 110, 222605 (2017).

51. Carbotte, J. P. Properties of boson-exchange superconductors. Rev. Mod. Phys. 62, 1027-1157 (1990).

52. Günel, H. Y. et al. Supercurrent in $\mathrm{Nb} / \mathrm{InAs}$-nanowire/Nb Josephson junctions. J. Appl. Phys. 112, 034316 (2012).

53. Angers, L. et al. Proximity de squids in the long-junction limit. Phys. Rev. B 77, 165408 (2008).

54. Kim, B.-K. et al. Strong superconducting proximity effects in PbS semiconductor nanowires. ACS Nano 11, 221-226 (2017).

55. Zhang, J. et al. Proximity effect induced superconductivity in $\mathrm{Nb} / \mathrm{Sb}_{2} \mathrm{Te}_{3}-$ nanoribbon/Nb junctions. Ann. Phys. 532, 2000273 (2020).

56. Octavio, M., Tinkham, M., Blonder, G. E. \& Klapwijk, T. M. Subharmonic energy-gap structure in superconducting constrictions. Phys. Rev. B 27, 6739-6746 (1983).

57. Flensberg, K., Hansen, J. B. \& Octavio, M. Subharmonic energy-gap structure in superconducting weak links. Phys. Rev. B 38, 8707-8711 (1988)

58. Cuevas, J. C., Hammer, J., Kopu, J., Viljas, J. K. \& Eschrig, M. Proximity effect and multiple Andreev reflections in diffusive superconductor-normalmetal-superconductor junctions. Phys. Rev. B 73, 184505 (2006).

59. Dubos, P. et al. Josephson critical current in a long mesoscopic S-N-S junction. Phys. Rev. B 63, 064502 (2001).

60. Frielinghaus, R. et al. Josephson supercurrent in $\mathrm{Nb} / \mathrm{InN}$-nanowire/ $\mathrm{Nb}$ junctions. Appl. Phys. Lett. 96, 132504 (2010).

61. Cuevas, J. C. \& Bergeret, F. S. Magnetic interference patterns and vortices in diffusive SNS junctions. Phys. Rev. Lett. 99, 217002 (2007).

62. Kulik, I. O. Macroscopic quantization and the proximity effect in SNS junctions. Sov. Phys. JETP 57, 1745-1759 (1969).

63. Haas, F. et al. Quantum interferometer based on GaAs/InAs core/shell nanowires connected to superconducting contacts. Semicond. Sci. Technol. 33, 064001 (2018).

64. Forschungszentrum Jülich GmbH. HNF-Helmholtz Nano Facility. J. Large Scale Facil. 3, A112 (2017)

\section{Acknowledgements}

We thank Peter Schüffelgen, Tobias Schmitt, and Michael Schleenvoigt for helpful discussions, Herbert Kertz for technical assistance and Stefan Trellenkamp and Lidia Kibkalo for assistance during prepration and characterization. Furthermore, we thank the Helmholtz Nano Facility (HNF) for help with devices fabrication in its clean room facilities ${ }^{64}$. This work was financially supported by the National Natural Science Foundation of China (Grant No. 62074058) and Shanghai Pujiang Program (20PJ1403600), the Deutsche Forschungsgemeinschaft (DFG, German Research Foundation) under the priority program SPP1666 "Topological Insulators", the Helmholtz Association the "Virtual Institute for Topological Insulators" (VITI, VHVI-511), by the Deutsche Forschungsgemeinschaft (DFG, German Research Foundation) under Germany's Excellence Strategy-Cluster of Excellence Matter and Light for Quantum Computing (ML4Q) EXC 2004/1-390 534 769, the National Key R\&D Program of China (Grant Nos. 2018YFB0406500 and 2017YFA0303403), and the Projects of Science and Technology Commission of Shanghai Municipality (Grant No. 18JC1412400).

\section{Author contributions}

J.Z., J.K., and D.R. performed the transport experiments, J.Z., A.R.J., J.K., D.R., and G.P. fabricated the samples, P.L.T. and J.G.L. fabricated the nanowires by VLS growth, M.L., A.R.J., J.Z., and J.G.L. performed the structural analysis. J.Z., A.R.J., H.L., Z.H., D.G., and T.S. wrote the paper. All authors contributed to the discussions.

\section{Funding}

Open Access funding enabled and organized by Projekt DEAL.

\section{Competing interests}

The authors declare no competing interests.

\section{Additional information}

Supplementary information The online version contains supplementary material available at https://doi.org/10.1038/s41467-021-21042-5.

Correspondence and requests for materials should be addressed to T.S.

Peer review information Nature Communications thanks Satyabrata Jit and the other, anonymous, reviewer(s) for their contribution to the peer review of this work.

Reprints and permission information is available at http://www.nature.com/reprints

Publisher's note Springer Nature remains neutral with regard to jurisdictional claims in published maps and institutional affiliations.

Open Access This article is licensed under a Creative Commons Attribution 4.0 International License, which permits use, sharing, adaptation, distribution and reproduction in any medium or format, as long as you give appropriate credit to the original author(s) and the source, provide a link to the Creative Commons license, and indicate if changes were made. The images or other third party material in this article are included in the article's Creative Commons license, unless indicated otherwise in a credit line to the material. If material is not included in the article's Creative Commons license and your intended use is not permitted by statutory regulation or exceeds the permitted use, you will need to obtain permission directly from the copyright holder. To view a copy of this license, visit http://creativecommons.org/ licenses/by/4.0/.

(C) The Author(s) 2021 